\title{
Influência do Apgar baixo na primeira triagem auditiva do neonato
}

\section{Influence of low Apgar on the first hearing screening of the neonate}

Daniela Polo Camargo da Silva1', Georgea Espindola Ribeiro², Jair Cortez Montovani²

‘Universidade Federal de Santa Catarina (UFSC) - Florianópolis (SC), Brasil.

"Faculdade de Medicina, Universidade Estadual Paulista "Júlio de Mesquita Filho" (UNESP) - Botucatu (SP), Brasil.

DOI: https://dx.doi.org/10.7322/abcshs.v44i2.1183

\section{RESUMO}

Introdução: Diversos são os indicadores de risco para deficiência auditiva. O índice de Apgar, quando menor que quatro no primeiro minuto e/ou menor que seis no quinto minuto de vida, é considerado um indicador de risco para surdez. Tais valores estão associados à ocorrência de asfixia, que é uma das causas mais comuns de lesão e morte celular e, desta forma, algum dano ao sistema auditivo pode ocorrer. Objetivo: Verificar a influência do indicador de risco para deficiência auditiva, Apgar baixo, na primeira avaliação auditiva do neonato. Métodos: Estudo clínico transversal, realizado em hospital público no período de janeiro de 2012 a dezembro de 2016. Os neonatos realizaram a avaliação audiológica por meio do exame de emissões otoacústicas evocadas por estímulo transiente (EOE-t). A relação entre Apgar baixo e "falha" nas EOE-t foi analisada por regressão logística simples. Resultados: Foram avaliados 6.301 neonatos, desses $15,82 \%(n=996)$ tinham indicadores de risco para deficiência auditiva e 6,57\% ( $n=415)$ obtiveram Apgar baixo. Apresentaram falha no exame de EOE-t, uni ou bilateral, 6,5\% ( $n=415)$ dos neonatos triados. Outros indicadores de risco aumentaram as chances de "falha" na EOE-t, porém o Apgar baixo, isoladamente, aumentou as chances de "falha" em neonatos com peso ao nascimento maior que $1.500 \mathrm{~g}(\mathrm{OR}: 1,58 ; p=0,02)$ e sem hemorragia peri-intraventricular (OR: 1,56; $p=0,01$ ). Conclusão: O índice de Apgar baixo foi o principal causador de "falha" na primeira avaliação auditiva dentre os neonatos com peso maior de $1.500 \mathrm{~g}$ e sem hemorragia peri-intraventricular.

Palavras-chave: triagem neonatal; indicador de risco; índice de Apgar; Audição.

\begin{abstract}
Introduction: There are several risk indicators for hearing loss. The Apgar score, when less than four in the first minute and/or less than six in the fifth minute of life, is considered an indicator of risk for hearing loss. Such values are associated with the occurrence of asphyxia, which is one of the most common causes of cell death and injury and, therefore, some damage to the auditory system may occur. Objective: To check the influence of risk indicator for hearing loss, low Apgar, in the first neonatal hearing evaluation. Methods: Cross-sectional study conducted in a public hospital from January 2012 to December 2016. The neonates underwent hearing evaluation by examining transient-evoked otoacoustic emissions (TEOAE). The relation between low Apgar and "refer" in the TEOAE was analyzed by simple logistic regression. Results: A total of 6,301 newborns were evaluated, of which $15.82 \%(n=996)$ had risk indicators for hearing loss and $6.57 \%(n=415)$ had with low Apgar. $6.5 \%(n=415)$ of the neonates screened showed failure of the EOE-t test, uni or bilateral. Others risk indicators increased the chances of "refer"; however, the presence of low Apgar alone increased the chances of "refer" in the TEOAE in newborns with birth weight greater than 1,500 $\mathrm{g}(\mathrm{OR}: 1.58 ; \mathrm{p}=0.02)$ and without peri-intraventricular hemorrhage (OR: 1.56; $p=0.01$ ). Conclusion: The low Apgar score was the mainly cause of "refer" on the first hearing evaluation among neonates with with birth weight greater than 1,500 $\mathrm{g}$ and without peri-intraventricular hemorrhage.
\end{abstract}

Keywords: neonatal screening; risk index; Apgar score; hearing.

Recebido em: 13/07/2018

Revisado em: 11/12/2018

Aprovado em: 20/12/2018

Autor para correspondência: Daniela Polo Camargo da Silva - Departamento de Fonoaudiologia, Universidade Federal de Santa Catarina - Campus Reitor João David Ferreira Lima, s/n - Trindade - CEP: 88040-900 - Florianópolis (SC), Brasil - E-mail: daniela-polo@uol.com.br

Conflito de interesses: nada a declarar. 


\section{INTRODUÇÃO}

A perda auditiva é dificilmente percebida nos primeiros meses de vida da criança. Se não detectada e tratada em tempo hábil, ela pode levar a um retardo importante do desenvolvimento cognitivo e intelectual, especialmente da fala, além de associar-se a sérios problemas emocionais e educacionais, tanto para a criança quanto para seus familiares ${ }^{1,2,3}$. Assim, justifica-se a realização da triagem auditiva neonatal para a identificação precoce da deficiência auditiva ${ }^{1}$.

Apesar da existência de diversos protocolos para utilização na detecção e diagnóstico precoce da deficiência auditiva, as emissões otoacústicas como primeiro método de avaliação é o mais frequentemente utilizado, devido a sua fácil e rápida aplicabilidade associada ao baixo custo financeiro, quando comparada ao potencial evocado auditivo de tronco encefálico ${ }^{4,5,6}$.

O Joint Committee on Infant Hearing (JCIH) aponta diversos indicadores de risco que são intimamente associados à deficiência auditiva, sendo estes revalidados ao longo dos anos por diversos estudos ${ }^{1,7,8}$. Em consequência das mudanças nos tipos de intervenções terapêuticas e das características da população admitida em Unidade de Terapia Intensiva (UTI), atualmente, tais indicadores de risco precisam ser revistos para comprovar se ainda são significativos para a deficiência auditiva ${ }^{9,10}$. A surdez causada por rubéola congênita, por exemplo, muito comum no século passado, nos dias de hoje quase não é observada em decorrência de medidas preventivas ${ }^{1,7,8}$.

Estudos atuais mostram a influência de indicadores de risco para deficiência auditiva em neonatos que foram internados em $\mathrm{UTI}^{11}$. Outros estudos também mostram uma maior ocorrência de perda auditiva em neonatos com síndromes genéticas e com história de ventilação mecânica maior que cinco dias 7 . O Apgar baixo, menor que quatro no primeiro minuto e/ou menor que seis no quinto minuto de vida, apresentou associação com a perda auditiva sensorioneural, independente do tempo de permanência na $\mathrm{UTI}^{8,11}$. Alguns estudiosos justificam a perda auditiva em neonatos que sofreram asfixia perinatal, devido uma diminuição do fluxo de sangue para a orelha interna provocando apoptose celular ${ }^{12}$.

Sabe-se que os neonatos admitidos em UTI que apresentam muitos indicadores de risco para a deficiência auditiva, têm altos índices de falha na primeira avaliação auditiva ${ }^{11,13}$.

Além disso, há a hipótese de que essas alterações auditivas ocorrem em decorrência de uma associação de vários indicadores de risco para a deficiência auditiva, assim como, também podem ser causadas por algum indicador em particular ${ }^{11,13}$.

A asfixia perinatal está comumente relacionada com várias condições médicas, de modo que é difícil estabelecer a asfixia absolutamente como a única causa específica de surdez $z^{14,15}$. O índice de Apgar é preditor para o diagnóstico da asfixia perinatal e sua avaliação, de forma isolada, nos resultados da triagem auditiva pode contribuir para o conhecimento do potencial desta condição na falha inicial, o que permitirá ter melhor conduta, acompanhamento e intervenção nos casos de deficiência auditiva ${ }^{14,15,16}$.
Portanto, o objetivo deste estudo foi verificar a influência do indicador de risco para deficiência auditiva, Apgar baixo, na primeira avaliação auditiva do neonato.

\section{MÉTODOS}

Estudo clínico transversal, realizado em hospital público do interior do estado de São Paulo. Os dados foram coletados de janeiro de 2012 a dezembro de 2016. O estudo foi submetido e houve aprovação do Comitê de Ética em Pesquisa local (processo $\left.\mathrm{n}^{\circ} 3395 / 09\right)$.

Foram encaminhados para triagem auditiva 6.477 neonatos, porém, 6.301 neonatos atenderam aos seguintes critérios de inclusão: a) ter nascido no local de realização do estudo, b) apresentar ou não indicador de risco para deficiência auditiva, c) ter avaliação otorrinolaringológica normal, d) ter sido atendido no programa de triagem auditiva neonatal no local de realização do estudo, e) ter o Termo de Consentimento Livre e Esclarecido assinado pelos responsáveis do neonato.

A triagem auditiva baseou-se no seguinte protocolo: emissões otoacústicas em todos os neonatos, seguida do PEATE (não automático) naqueles que apresentaram risco para a deficiência auditiva e nos que apresentaram "falha" no reteste das emissões, que foi realizado em dia e local distinto. Portanto, o primeiro teste com emissões otoacústicas foi selecionado para o estudo por ser crucial na determinação de qual procedimento audiológico subsequente foi necessário na rotina do serviço.

O índice de Apgar verifica o estado clínico do recém-nascido e também identifica aqueles que necessitam de assistência, pois avalia riscos de uma asfixia perinatal ${ }^{16}$. São cinco os seus critérios avaliados: frequência cardíaca, respiração, tônus muscular, irritabilidade reflexa e coloração da pele. Cada critério recebe um valor que pode variar de 0 a 2 e quanto maior a pontuação melhor foi às condições ao nascimento. Esta avaliação é realizada no primeiro, no quinto e no décimo minuto de vida, pelo médico neonatologista ${ }^{16}$. Quando os valores obtidos são menores que quatro no primeiro minuto e/ou menor que seis no quinto minuto de vida, são considerados indicadores de risco para deficiência auditiva ${ }^{1}$.

O exame de emissões otoacústicas evocadas por estímulo transiente (EOE-t) foi realizado com o lactente em estado de sono natural no colo da mãe. Todos os neonatos apresentavam idade maior que 48 horas de vida, no momento da avaliação. O teste foi realizado em um único momento, com o equipamento OtoRead/Interacoustics:

Os critérios para os resultados "PASSA/FALHA" empregados neste estudo foram os referidos pelo protocolo do equipamento sendo eles: estímulo clique na intensidade de $83 \mathrm{dBSPL}$ (decibel sound pressure level); nas bandas de frequência de $1.500 \mathrm{~Hz}$ a $4.000 \mathrm{~Hz}$. Para o resultado "PASSA" as emissões se apresentavam numa relação sinal/ruído de $6 \mathrm{~dB}$ (decibel) em pelo menos três bandas de frequências sequentes. 
As variáveis foram classificadas em: a) exposição (presença/ ausência de Apgar baixo); b) confusão (idade gestacional ao nascimento e presença de outro indicador de risco para deficiência auditiva, além do Apgar baixo) e c) desfecho ("falha" nas EOE-t, em pelo menos uma orelha).

\section{Análise Estatística}

Para análise da associação entre índice de Apgar baixo e a chance de "falha" nas emissões otoacústicas foram ajustados modelos de regressão logística simples, em cada nível de variação: idade gestacional, peso ao nascer, hemorragia peri-intraventricular, permanência em UTI, ventilação mecânica e uso de ototóxicos.

As associações foram consideradas estatisticamente significantes se $\mathrm{p}<0,05$. Análise com o software SP5s v21.0.

\section{RESULTADOS}

Dentre os 6.477 neonatos que realizaram a triagem auditiva durante o período do estudo, 6.301 atenderam aos critérios de inclusão, com idade média de 0,7 meses (mínimo de 0 e máximo de 3 meses). Desses, 415 (6,5\%) neonatos tiveram Apgar baixo. A caracterização da amostra encontra-se na Tabela 1. Os indicadores de risco mais frequentes entre os neonatos que "falharam" na triagem auditiva foram permanência em UTI maior que cinco dias, prematuridade e Apgar baixo.

Tabela 1: Caracterização de todos os neonatos avaliados

\begin{tabular}{|c|c|c|}
\hline Variável & $\mathbf{n}$ & $\%$ \\
\hline \multicolumn{3}{|l|}{ Apgar baixo } \\
\hline Não & 5886 & 93,5 \\
\hline Sim & 415 & 6,5 \\
\hline \multicolumn{3}{|c|}{ Falha nas Emissões Otoacústicas ( $n=6.301$ ) } \\
\hline Não & 5886 & 93,5 \\
\hline Sim & 415 & 6,5 \\
\hline \multicolumn{3}{|l|}{ Idade gestacional } \\
\hline Recém-nascido a termo & 5090 & 80,78 \\
\hline Recém-nascido pré-termo & 1211 & 19,22 \\
\hline \multicolumn{3}{|l|}{ Peso ao nascer } \\
\hline$\geq 1.500 \mathrm{~g}$ & 6063 & 96,22 \\
\hline$<1.500 \mathrm{~g}$ & 238 & 3,78 \\
\hline \multicolumn{3}{|l|}{ Hemorragia peri-intraventricular } \\
\hline Não & 6245 & 99,2 \\
\hline Sim & 56 & 0,8 \\
\hline \multicolumn{3}{|l|}{ Permanência em UTI } \\
\hline Não & 5877 & 93,3 \\
\hline Sim & 424 & 6,7 \\
\hline \multicolumn{3}{|l|}{ Ventilação mecânica } \\
\hline Não & 6066 & 96,3 \\
\hline Sim & 235 & 3,7 \\
\hline \multicolumn{3}{|l|}{ Uso de antibiótico } \\
\hline Não & 6143 & 97,5 \\
\hline Sim & 158 & 2,5 \\
\hline
\end{tabular}

UTI: Unidade de Terapia Intensiva
A análise preliminar das características desta amostra indicou que os seguintes indicadores de risco para a deficiência auditiva, além do Apgar baixo, se relacionam com o aumento de chance em "falhar" na triagem auditiva: prematuridade extrema $\mathrm{OR}=3,41$ (IC95\% 2,28-5,11), peso ao nascimento $<1.500$ g OR=3,06 (IC95\% 2,15-4,37), ocorrência de hemorragia peri-intraventricular OR=2,39 (IC95\% 1,12-5,09), permanência em UTI OR=2,51 (IC95\% 1,87-3,37), uso de ventilação mecânica OR=2,07 (IC95\% 1,39-3,10) e uso de medicamentos ototóxicos OR=3,20 (IC95\% 2,10-4,88). Portanto, a relação entre Apgar baixo e "falha" na triagem auditiva foi estimada com correção para essas variáveis.

O Apgar baixo aumentou as chances de "falha" na triagem auditiva, independentemente de outros indicadores de risco coexistentes em neonatos que tiveram peso ao nascimento maior de $1.500 \mathrm{~g} \mathrm{e}$ em neonatos sem hemorragia peri-intraventricular (Tabela 2).

\section{DISCUSSÃO}

Esse estudo foi realizado em um hospital público, que atende principalmente gestantes de alto risco e, consequentemente, é onde as chances de complicações perinatais e deficiência auditiva são potencialmente maiores ${ }^{17}$.

A identificação de qual indicador de risco para deficiência auditiva que mais afetou o primeiro exame auditivo do neonato não é uma tarefa fácil. A presença dos indicadores de risco, peso menor que $1.500 \mathrm{~g}$ ao nascimento, permanência em UTI maior

Tabela 2: Modelo de regressão logística para a chance de "falha" em função do índice de Apgar baixo"

\begin{tabular}{|c|c|c|c|c|c|}
\hline Modelo & Idade gestacional & $\beta$ & p & OR & IC95\% \\
\hline 1 & Recém-nascido a termo & 0,10 & 0,721 & 1,11 & $0,63-1,97$ \\
\hline 2 & $\begin{array}{l}\text { Recém-nascido } \\
\text { pré-termo }\end{array}$ & 0,41 & 0,138 & 1,51 & $0,88-2,60$ \\
\hline \multirow[t]{2}{*}{3} & $\begin{array}{l}\text { Recém-nascido } \\
\text { pré-termo extremo }\end{array}$ & $-0,24$ & 0,558 & 0,78 & $0,34-1,78$ \\
\hline & Peso ao nascer & & & & \\
\hline 4 & $\geq 1.500 \mathrm{~g}$ & 0,46 & $0,022^{*}$ & 1,58 & $1,07-2,33$ \\
\hline \multirow[t]{2}{*}{5} & $<1.500 \mathrm{~g}$ & $-0,50$ & 0,195 & 0,61 & $0,29-1,29$ \\
\hline & $\begin{array}{l}\text { Hemorragia } \\
\text { peri-intraventricular }\end{array}$ & & & & \\
\hline 6 & Não & 0,44 & $0,014^{*}$ & 1,56 & $1,09-2,22$ \\
\hline \multirow[t]{2}{*}{7} & Sim & 0,34 & 0,660 & 1,40 & $0,31-6,27$ \\
\hline & Permanência em UTI & & & & \\
\hline 8 & Não & 0,11 & 0,656 & 1,12 & $0,68-1,83$ \\
\hline \multirow[t]{2}{*}{9} & Sim & 0,24 & 0,404 & 1,27 & $0,72-2,24$ \\
\hline & Ventilação mecânica & & & & \\
\hline 10 & Não & 0,34 & 0,096 & 1,41 & $0,94-2,10$ \\
\hline \multirow[t]{2}{*}{11} & Sim & 0,37 & 0,354 & 1,45 & $0,66-3,18$ \\
\hline & Uso de ototóxicos & & & & \\
\hline 12 & Não & 0,37 & 0,062 & 1,44 & $0,98-2,11$ \\
\hline 13 & Sim & 0,05 & 0,912 & 1,05 & $0,45-2,46$ \\
\hline
\end{tabular}

OR: odds ratio; IC: índice de correlação; UTI: Unidade de Terapia Intensiva* ${ }^{\star}<0,05$ 
que cinco dias, uso de ventilação mecânica, uso de medicamentos ototóxicos e ocorrência de prematuridade, mostraram, neste estudo, uma relação direta com maior índice de "falha" na primeira avaliação auditiva ${ }^{18,19}$.

Este resultado confirma a ideia de vários fatores contribuindo para a "falha" no primeiro exame $e^{7,910,17}$. Após a correção estatística dos dados por estas variáveis, o Apgar baixo mostrou aumentar as chances de "falha" na avaliação inicial de neonatos com peso maior que $1.500 \mathrm{~g}$ ao nascimento e naqueles que não tinham hemorragia peri-intraventricular.

O Apgar baixo, além de auxiliar na determinação da ocorrência da asfixia perinatal, mostrou que as células ciliadas externa da cóclea são extremamente sensíveis a baixa oxigenação e dependendo da gravidade pode trazer danos irreversíveis à cóclea comprometendo os resultados das EOE-t e, consequentemente, a acuidade auditiva desses neonatos o que causa o aumento do número de "falha" na TANU ${ }^{14}$

O Apgar baixo foi associado com aumento do número de falhas na triagem neonatal nos estudos de Regina et al. ${ }^{20}$, Gouri et al. ${ }^{21}$ e Maqbool et al. ${ }^{22}$ em uma frequência de 10,52\%, 16,7\% e 12,5\%, respectivamente e concluíram que o índice de Apgar $\leq 5$ no primeiro minuto foi um fator de risco significativo para o comprometimento da audição.

Este achado sugere que, mesmo com a melhora assistencial imediata no período neonatal, ao longo dos anos, o Apgar baixo ainda influencia a "falha" na avaliação inicial".

É válida a reflexão de que esse achado de maior ocorrência de "falha", nesta primeira avaliação, não implica em maior número de casos com deficiência auditiva, por isso a reavaliação dos neonatos, como também o uso de medidas eletrofisiológicas, irá auxiliar no conhecimento do real efeito deste indicador de risco no sistema auditivo ${ }^{8,23}$.
A asfixia perinatal, evidenciada pelo índice de Apgar, está diretamente relacionada ao dano coclear, de caráter reversível, caso a oxigenação normal seja rapidamente retomada e, de caráter irreversível, em casos de asfixia persistente, causando lesões na condução do nervo auditivo ${ }^{14,15,16,17}$.

Uma diminuição da atividade das células ciliadas externas nos primeiros dias de vida em neonatos que sofreram comprometimento do sistema nervoso central e asfixia perinatal ${ }^{15}$, reforça a importância de se realizar emissões otoacústicas nestes $\operatorname{casos}^{5,12}$. Há ainda estudos que demonstram diminuição da amplitude de resposta no exame de emissões, sem necessariamente causar falha, nestas situações ${ }^{12}$.

Além disso, a falta de oxigenação pode provocar perda auditiva sensorioneural em frequências altas, o que pode ter impacto significativo no desenvolvimento de fala e linguagem, uma vez que é nessa região que se concentra o espectro de audibilidade da maioria das consoantes ${ }^{24,25}$.

Atribui-se o índice elevado de Apgar baixo nesse estudo ao cenário em que ele foi desenvolvido, já que se trata de um hospital referência em atendimento à gestante de alto risco.

Neste contexto, o conhecimento do Apgar baixo afetar o primeiro exame auditivo do neonato com peso maior de $1.500 \mathrm{~g}$ ou sem hemorragia peri-intraventricular, pode favorecer uma conduta cautelosa dos profissionais envolvidos na saúde materno-infantil, devendo o neonato ser encaminhado para avaliação audiológica completa ${ }^{1,26}$. Quando houver confirmação da perda auditiva sensorioneural, o diagnóstico diferencial coclear/retrococlear possibilitará que o neonato receba o tratamento mais adequado ${ }^{1,26}$.

Em conclusão, o Apgar baixo foi o principal causador de "falha" na primeira avaliação auditiva, por EOE-t, independentemente de outros indicadores de risco coexistentes, em neonatos com peso maior de $1.500 \mathrm{~g}$ ou sem hemorragia peri-intraventricular.

\section{REFERÊNCIAS}

1. Joint Committee on Infant Hearing. Year 2007 position statement: principles and guidelines for early hearing detection and intervention programs. Pediatrics. 2007; 120(4):898-921.

http://dx.doi.org/10.1542/peds.2007-2333

2. Rai N, Thakur N. Universal screening of newborns to detect hearing impairment-is it necessary? Int J Pediatr Otorhinolaryngol. 2013;77(6): 1036-41

http://dx.doi.org/10.1016/j.ijporl.2013.04.006

3. Alvarenga KF, Araújo ES, Melo TM, Martinez MAN, Bevilacqua MC. Questionnaire for monitoring auditory and language development in the first year. CoDAS. 2013;25(1):16-21.

http://dx.doi.org/10.1590/S2317-17822013000100004

4. Lim HW, Kim EAR, Chung JW. Audiological follow-up results after newborn hearing screening program. Clin Exp Otorhinolaryngol. 2012:5(2):57-61.

http://dx.doi.org/10.3342/ceo.2012.5.2.57
5. Amini E, Farahani ZK, Samani MR, Hamedi H, Zamini A, Yazdi $A K$, et al. Assessment of hearing loss by OAE in asphyxiated newborns. Iran Red Crescent Med J. 2014:16(1):e6812. https://doi.org/10.1055/s-0038-1648217

6. Lima MC, Rossi TR, Françozo Mde F, Collela-Santos MF, Correa $\mathrm{CR}$. Analysis of neonatal hearing screening program performed on an outpatient basis: Analysis of an outpatient hearing screening program. Int J Pediatr Otorhinolaryngol. 2015;79(12):2227-33. http://doi.org/10.1016/.j.jporl.2015.10.009

7. Kiatchoosakun P, Suphadun W, Jirapradittha J, Yimtae K, Thanawirattananit P. Incidence and risk factors associated with hearing loss in high-risk neonates in Srinagarind Hospital. J Med Assoc Thai. 2012;95(1):52-7.

8. Cavalcanti HG, Melo LPF, Buarque LFSFP, Guerra RO. Overview of newborn hearing screening programs in Brazilian maternity hospitals. Braz J Otorhinolaryngol. 2014:80:346-53 http://dx.doi.org/10.1016/j.bjorl.2014.05.005 
9. Wróbel MJ, Greczka G, Szyfter W. The risk factor profile of children covered by Polish universal neonatal hearing screening program and its impact on hearing loss incidence. Int $\mathrm{J}$ Pediatr Otorhinolaryngol. 2014;78(2):209-13.

https://dx.doi.org/10.1016/j.ijporl.2013.10.052

10. Beswick R, Driscoll C, Kei J, Khan A, Glennon S. Which risk factors predict postnatal hearing loss in children? J Am Acad Audiol. 2013;24(3):205-13

https://dx.doi.org/10.3766/jaaa.24.3.6

11. Colella-Santos MF, Hein TAD, Souza GL, Amaral MIR, Casali RL. Newborn Hearing Screening and Early Diagnostic in the NICU. 2014; 2014:1-11

http://dx.doi.org/10.1155/2014/845308

12. Ribeiro GE, Silva DPC, Montovani JC. Transient evoked otoacoustic emissions and auditory brainstem response in infants with perinatal asphyxia. Int J Pediatr Otorhinolaryngol. 2016;89:136-9. http://dx.doi.org/10.1016/j.ijporl.2016.08.009

13. Oliveira JS, Rodrigues LB, Aurélio FS, Silva VB. Risk factors and prevalence of newborn hearing loss in a private health care system of Porto Velho, Northern Brazil. Rev Paul Pediatr. 2013;31(3):299-305

http://dx.doi.org/10.1590/S0103-05822013000300005

14. Daniel SJ, Mcintosh M, Akinpelu OV, Rohlicek CV. Hearing outcome of early postnatal exposure to hypoxia in Sprague-Dawley rats. $J$ Laryngol Otol. 2014;128(4):331-5. http://dx.doi.org/10.1017/S002221511300265X

15. Boskabadi H, Omidian M, Tavallai S, Mohammadi S, Parizadeh M, Mobarhan MG, et al. Serum Hsp70 antigen: early diagnosis marker in perinatal asphyxia. Iran J Pediatr. 2015;25(2):e381. http://dx.doi.org/10.5812/ijp.381

16. Medeiros TKS, Dobre M, Silva DMB, Brateanu A, Baltatu OC, Campos LA. Intrapartum fetal heart rate: a possible predictor of neonatal acidemia and APGAR Score. Front Physiol. 2018;9:1489. https://dx.doi.org/10.3389/fphys.2018.01489

17. Oliveira DC, Mandú ENT. Women with high-risk pregnancy: experiences and perceptions of needs and care. Esc Anna Nery. 2015;19(1):93-101. http://dx.doi.org/10.5935/1414-8145.20150013
18. Silva DP, Lopez PS, Ribeiro GE, Luna MO, Lyra JC, Montovani JC. The importance of retesting the hearing screening as an indicator of the real early hearing disorder. Braz J Otorhinolaryngol. 2015; 81:363-7.

http://dx.doi.org/10.1016/j.bjorl.2014.07.019

19. Januário GC, Lemos SMA, Friche AAL, Alves CRL. Quality indicators in a newborn hearing screening service. Braz $J$ Otorhinolaryngol. 2015;81(3):255-63. http://dx.doi.org/10.1016/j.bjorl.2014.08.008

20. Maqbool M, Najar BA, Gattoo I, Chowdhary J. Screening for hearing impairment in high risk neonates: a hospital based study. J Clin Diagn Res. 2015;9(6):18-21. http://dx.doi.org/10.7860/JCDR/2015/14509.6104

21. Gouri ZUH, Sharma D, Berwal PK, Pandita A, Pawar S. Hearing impairment and its risk factors by newborn screening in northwestern India. Matern Health Neonatol Perinatol. 2015;1:17. https://dx.doi.org/10.1186/s40748-015-0018-1

22. Regina M, Moideen SP, Mohan M, Mohammed MTP, Afroze KHM. Audiological screening of high risk infants and prevalence of risk factors. Int J Contemp Pediatr 2017;4(2):507-11. http://dx.doi.org/10.18203/2349-3291.ijcp20170699

23. Rodrigues PAL, Nardez TMB, Espindola MM, Gomes KCC, Silva $\mathrm{BL}$. Comparison of two newborn hearing screening protocols with distinct reference criteria of distinct pass and failure. Rev CEFAC. 2016;18(4):876-80.

http://dx.doi.org/10.1590/1982-021620161842816

24. Leite JN, Silva VS, Buzo BC. Otoacoustic emissions in newborns with mild and moderate perinatal hypoxia. Codas. 2016;28(2):93-8.

http://dx.doi.org/10.1590/2317-1782/20162015086

25. Jiang ZD, Zang Z, Wilkinson AR. Cochlear function in 1-year-old term infants born with hypoxia-ischaemia or low Apgar scores. $J$ Paediatr Child Health. 2012;48(2):160-5. http://dx.doi.org/10.1111/j.1440-1754.2011.02066.x

26. Lewis DR, Marone SAM, Mendes BCA, Cruz OLM, Nóbrega M. Comitê multiprofissional em saúde auditiva COMUSA. Braz J Otorhinolaryngol. 2010;76(1):121-8 http://dx.doi.org/10.1590/S1808-86942010000100020 